\title{
Analysis of constrained theories without use of primary constraints.
}

\author{
A.A. Deriglazov* \\ Dept. de Matematica, ICE, Universidade Federal de Juiz de Fora, \\ MG, Brasil.
}

\begin{abstract}
It is shown that the Dirac approach to Hamiltonization of singular theories can be slightly modified in such a way that primary Dirac constraints do not appear in the process. According to the modified scheme, Hamiltonian formulation of singular theory is first order Lagrangian formulation, further rewritten in special coordinates.
\end{abstract}

\section{Introduction}

Let us consider Lagrangian theory with the action and the equations of motion being

$$
\begin{gathered}
S=\int d \tau \bar{L}\left(q^{A}, \dot{q}^{A}\right), \quad A=1,2, \cdots[A], \\
\left(\frac{\partial \bar{L}}{\partial \dot{q}^{A}}\right) \cdot-\frac{\partial \bar{L}}{\partial q^{A}}=0 .
\end{gathered}
$$

It is supposed that the theory is singular

$$
\operatorname{rank} \frac{\partial^{2} \bar{L}}{\partial \dot{q}^{A} \partial \dot{q}^{B}}=[i]<[A] .
$$

According to Dirac [1], Hamiltonian formulation of the theory is obtained as follow. First step of Hamiltonization procedure is to define equations for the momenta $p_{A}: p_{A}=\frac{\partial \bar{L}}{\partial \dot{q}^{A}}$. The latter can be

*alexei@ice.ufjf.br On leave of absence from Dept. Math. Phys., Tomsk Polytechnical University, Tomsk, Russia. 
considered as algebraic equations for determining of velocities $\dot{q}^{A}$. According to the rank condition (3), $[i]$ equations can be resolved for $\dot{q}^{i}$ and then substituted into the remaining ones. By construction, the resulting equations do not depend on $\dot{q}^{A}$ and are called primary constraints $\Phi_{\alpha}(q, p)$ of the Hamiltonian formulation. The equations $p_{A}=\frac{\partial \bar{L}}{\partial \dot{q}^{A}}$ are then equivalent to the following system

$$
\begin{aligned}
& \dot{q}^{i}=v^{i}\left(q^{A}, p_{i}, \dot{q}^{\alpha}\right), \\
& \Phi_{\alpha}\left(q^{A}, p_{\alpha}, p_{i}\right)=0 .
\end{aligned}
$$

On second step one introduces an extended phase space with the coordinates $\left(q^{A}, p_{A}, v_{\alpha}\right)$. By definition, Hamiltonian formulation of the theory (2) is the following system of equations on this space

$$
\begin{gathered}
\dot{q}^{A}=\left\{q^{A}, H\right\}, \dot{p}_{A}=\left\{p_{A}, H\right\}, \\
\Phi_{\alpha}\left(q^{A}, p_{B}\right)=0,
\end{gathered}
$$

where $\{$,$\} is the Poisson bracket, and it was denoted$

$$
\begin{gathered}
H\left(q^{A}, p_{A}, v^{\alpha}\right)=H_{0}\left(q^{A}, p_{j}\right)+v^{\alpha} \Phi_{\alpha}\left(q^{A}, p_{B}\right), \\
H_{0}=\left.\left(p_{i} \dot{q}^{i}-\bar{L}+\dot{q}^{\alpha} \frac{\partial \bar{L}}{\partial \dot{q}^{\alpha}}\right)\right|_{\dot{q}^{i} \rightarrow v^{i}\left(q^{A}, p_{j}, \dot{q}^{\alpha}\right)} .
\end{gathered}
$$

It is known [2] that the formulations (2) and (6) are equivalent.

Aim of this part of the Dirac procedure is, in fact, to represent system of differential equations (2) in the normal form (with derivatives of variables separated on the l.h.s. of equations). Adopting this point of view, we demonstrate below that Eq.(5), as well as the variables $p_{\alpha}$, are not necessary in the process. In Sect. 2 we formulate Hamiltonization procedure for the theory (11)-(3) without use of the momenta $p_{\alpha}$. As a consequence, the primary constraints (15) do not appear in the formulation. Concerning to Eq.(44), it has simple interpretation [3] as (invertible) change of variables on configuration-

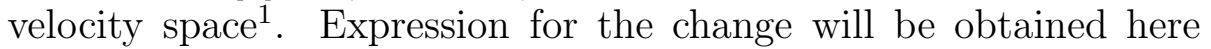
as sufficient condition for conversion of equations of motion (see Eq.(17) below) into the normal form. Equivalence with the Dirac procedure is shown in Sect.3. Some useful identities are collected in the Appendix.

\footnotetext{
${ }^{1}$ In this respect, the formulation presented below is more close to the case of nonsingular theory, where transition from the Lagrangian to the Hamiltonian formalism is change of variables.
} 


\section{Hamiltonization without use of primary con- straints}

We present here modification of standard Hamiltonization procedure $[1,2]$ which do not implies appearance of the primary constraints in the formalism.

1 Step. System of differential equations of second order (2) can be rewritten in a first order form. To achieve this, one introduces configuration-velocity space with the coordinates $\left(q^{A}, v^{A}\right)$. The initial system and the following one are equivalent ${ }^{2}$

$$
\dot{q}^{A}=v^{A}, \quad \bar{M}_{A B} \dot{v}^{B}=\bar{K}_{A} .
$$

Here it was denoted

$$
\bar{M}_{A B}(q, v) \equiv \frac{\partial^{2} \bar{L}(q, v)}{\partial v^{A} \partial v^{B}}, \quad \bar{K}_{A}(q, v) \equiv \frac{\partial \bar{L}}{\partial q^{A}}-\frac{\partial^{2} \bar{L}}{\partial v^{A} \partial q^{B}} v^{B} .
$$

We use symbols with bar to denote functions on the configurationvelocity space: $\bar{A} \equiv \bar{A}\left(q^{A}, v^{B}\right)$, and symbols without bar for functions on extended phase space (see below)

$$
A=\left.A\left(q^{A}, p_{j}, v^{\alpha}\right) \equiv \bar{A}\left(q^{A}, v^{\alpha}, v^{i}\right)\right|_{v^{i}\left(q^{A}, p_{j}, v^{\alpha}\right)} .
$$

Without loss of generality [2], we suppose that the variables $q^{A}$ has been enumerated in a way adjusted with Eq.(3), then

$$
\begin{gathered}
\bar{M}_{A B}=\left(\begin{array}{cc}
\bar{M}_{i j} & \bar{M}_{i \beta} \\
\bar{M}_{\alpha j} & \bar{M}_{\alpha \beta}
\end{array}\right), \\
A=(i, \alpha), \quad i=1,2, \cdots[i], \quad \alpha=1,2, \cdots[\alpha]=[A]-[i], \\
\operatorname{rank} \bar{M}_{A B}=[i], \quad \operatorname{det} \bar{M}_{i j} \neq 0 .
\end{gathered}
$$

Inverse matrix for $\bar{M}_{i j}$ is denoted $\tilde{\bar{M}}^{i j}$, one has $\bar{M}_{i j} \tilde{\bar{M}}^{j k}=\delta_{i}{ }^{k}$.

2 Step. According to the rank condition, $[i]$ equations among (91) can be resolved in relation of $\dot{v}^{i}: \dot{v}^{i}=\tilde{\bar{M}}^{i j}\left(K_{j}-\bar{M}_{j \alpha} \dot{v}^{\alpha}\right)$ and then substituted into remaining $[\alpha]$ equations with the result being $\left[\bar{M}_{\alpha \beta}-\bar{M}_{\alpha i} \tilde{\bar{M}}^{i j} \bar{M}_{j \beta}\right] \dot{v}^{\beta}=\bar{K}_{\alpha}-\bar{M}_{\alpha i} \tilde{\bar{M}}^{i j} \bar{K}_{j}$. It must be $\operatorname{rank}\left[\bar{M}_{\alpha \beta}-\right.$ $\left.\bar{M}_{\alpha i} \tilde{\bar{M}}^{i j} \bar{M}_{j \beta}\right]=0$ (if not, one would be able to resolve the equations

${ }^{2}$ The systems [2] and (9] are equivalent in the usual sense [2]: a) if $q_{0}(\tau), v_{0}(\tau)$ is solution of [9], then $q_{0}(\tau)$ is solution of [2] b) if $q_{0}(\tau)$ is solution of [2] then $q_{0}(\tau), p_{0}(\tau) \equiv \dot{q}_{0}$ is solution of 9]. 
in relation to some of $\dot{v}^{\alpha}$, in contradiction with (3) ). Then one has the identity

$$
\bar{M}_{\alpha \beta}-\bar{M}_{\alpha i} \tilde{\bar{M}}^{i j} \bar{M}_{j \beta}=0 .
$$

As a consequence, the following quantities

$$
\bar{C}_{\alpha}{ }^{A} \equiv\left(-\bar{M}_{\alpha j} \tilde{\bar{M}}^{j i}, \delta_{\alpha}^{\beta}\right)
$$

form a basis on space of null-vectors of the matrix $\bar{M}_{A B}$ (see also $[3])$

$$
\bar{C}_{\alpha}{ }^{A} \bar{M}_{A B}=0
$$

In the result, our equations (9) acquire the equivalent form

$$
\begin{gathered}
\dot{q}^{A}=v^{A}, \\
\dot{v}^{i}=\tilde{\bar{M}}^{i j}\left(\bar{K}_{j}-\bar{M}_{j \alpha} \dot{v}^{\alpha},\right) \\
\bar{K}_{\alpha}-\bar{M}_{\alpha i} \tilde{\bar{M}}^{i j} \bar{K}_{j} \equiv \bar{C}_{\alpha}{ }^{A} \bar{K}_{A}=0,
\end{gathered}
$$

where the system (18) is an algebraic one, (also, it may contain functionally dependent equations).

3 Step. Eq. (17) is not in the normal form. Then one asks on existence of special coordinates $\left(\tilde{q}^{A}, \tilde{v}^{A}\right)$ on $\left(q^{A}, v^{A}\right)$-space which reduce the system to the normal form. We demonstrate here the following statement.

For singular theory (11)-(3) there is exist invertible change of variables of the form

$$
\left(\begin{array}{c}
q^{A} \\
v^{\alpha} \\
v^{i}
\end{array}\right) \leftrightarrow\left(\begin{array}{c}
q^{A} \\
v^{\alpha} \\
p_{i}
\end{array}\right), \quad v^{i}=v^{i}\left(q^{A}, p_{j}, v^{\alpha}\right), \quad \operatorname{rank} \frac{\partial v^{i}}{\partial p_{j}}=[i],
$$

which reduces the system (17) to the normal form.

To make a proof, let us write Eq.(17) in terms of new variables (see our notations (11])

$$
M_{i j} \frac{\partial v^{i}}{\partial p_{k}} \dot{p}_{k}+\left(M_{i j} \frac{\partial v^{j}}{\partial v^{\alpha}}+M_{i \alpha}\right) \dot{v}^{\alpha}=K_{i}-M_{i j} \frac{\partial v^{j}}{\partial q^{A}} v^{A} .
$$


It reduces to the normal form if the functions $v^{i}$ obey the equations (note that $M_{i j}$ is the only invertible matrix at hand)

$$
\frac{\partial v^{i}}{\partial p_{j}}=\tilde{M}^{i j}, \quad \frac{\partial v^{i}}{\partial v^{\alpha}}=-\tilde{M}^{i j} M_{j \alpha} .
$$

These equations determine $v^{i}$ modulo of an arbitrary function $A\left(q^{A}\right)$, the latter will be omitted in what follow. Suppose that there is exist a solution of Eq.(21), and let $p_{i}\left(q^{A}, v^{A}\right)$ be inverse transformation. Expression for the latter can be easily obtained. Actually, using Eq.(21), derivatives of the identity $v^{i}\left(q^{A}, p_{i}\left(q^{A}, v^{A}\right), v^{\alpha}\right) \equiv v^{i}$ can be written in the form $\frac{\partial p_{i}}{\partial v^{j}}=\bar{M}_{i j}=\frac{\partial^{2} \bar{L}}{\partial v^{i} \partial v^{j}}, \quad \frac{\partial p_{i}}{\partial v^{\alpha}}=\bar{M}_{i \alpha}=\frac{\partial^{2} \bar{L}}{\partial v^{\alpha} \partial v^{i}}$. Solution of the system gives expression for $v^{i}$ in implicit form

$$
p_{i}=\frac{\partial \bar{L}\left(q^{A}, v^{i}, v^{\alpha}\right)}{\partial v^{i}} .
$$

It is necessary condition which turns out to be a sufficient condition also. Actually, Eq.(22) is invertible due to Eq.(3), and defines some function $v^{i}\left(q^{A}, p_{j}, v^{\alpha}\right)$. Then derivatives of the identity $\left.p_{i} \equiv \frac{\partial \bar{L}}{\partial v^{i}}\right|_{v^{i}}$ imply immediately the equations (21) (see also Appendix).

Curiously enough, the reasoning works for non singular theory also. To find normal form for non singular equations $\bar{M}_{A B} \dot{v}^{B}=\bar{K}_{A}$, one applies the inverse matrix $\tilde{\bar{M}}_{A B}$. Equivalently, one can search for a change $v^{A}\left(q^{A}, p_{A}\right)$ which reduces the system to the normal form. It gives the standard Hamiltonian formulation of the theory.

4 Step. Let us rewrite the equations (16)-(18) in the coordinates (19). Properties of the transformation $v^{i}=v^{i}\left(q^{A}, p_{j}, v^{\alpha}\right)$ are presented in the Appendix. In particular, it turns out to be linear on $v^{\alpha}$, and has the representation

$$
v^{i}\left(q^{A}, p_{j}, v^{\alpha}\right)=\frac{\partial H_{R}}{\partial p_{i}}
$$

where

$$
H_{R} \equiv H_{0}\left(q^{A}, p_{j}\right)-f_{\alpha}\left(q^{A}, p_{j}\right) v^{\alpha},\left.\quad f_{\alpha} \equiv \frac{\partial \bar{L}}{\partial v^{\alpha}}\right|_{v^{i}},
$$

and $H_{0}$ coincides with the Hamiltonian (8) of the Dirac scheme. Moreover, in the coordinates (19) all the quantities of the first order Lagrangian formulation can be rewritten in terms of the functions 
$H_{0}$ and $f_{\alpha}$, see Appendix. Using Eqs.(15), (23), (24), (34), (42), the system (16)-(18) can be identically rewritten in the form

$$
\begin{gathered}
\dot{q}^{\alpha}=v^{\alpha} \\
\dot{q}^{i}=\frac{\partial H_{R}}{\partial p_{i}}, \quad \dot{p}_{i}=-\frac{\partial H_{R}}{\partial q^{i}} \\
\triangle_{\alpha \beta}\left(q^{A}, p_{j}\right) v^{\beta}+H_{\alpha}\left(q^{A}, p_{j}\right)=0 .
\end{gathered}
$$

Here it was denoted

$$
\triangle_{\alpha \beta}=\left\{f_{\alpha}, f_{\beta}\right\}_{R}-\left(\frac{\partial f_{\alpha}}{\partial q^{\beta}}-\frac{\partial f_{\beta}}{\partial q^{\alpha}}\right), \quad H_{\alpha}=-\left\{f_{\alpha}, H_{0}\right\}_{R}-\frac{\partial H_{0}}{\partial q^{\alpha}},
$$

and $\{,\}_{R}$ is reduced Poisson bracket (i.e. the Poisson bracket on $q^{i}, p_{i^{-}}$subspace). For any functions $A, B$ of the variables $\left(q^{A}, p_{i}, v^{\alpha}\right)$ it is defined by

$$
\{A, B\}_{R}=\frac{\partial A}{\partial q^{i}} \frac{\partial B}{\partial p_{i}}-\frac{\partial A}{\partial p_{i}} \frac{\partial B}{\partial q^{i}} .
$$

It finishes first stage of the Hamiltonization procedure. Differential equations are presented in the normal form (26). One notes also that algebraic equations (27) turn out to be linear on $v^{\alpha}$. They coincide with the Dirac second stage system (for revealing of secondary constraints): $\left\{\Phi_{\alpha}, H\right\}=0$ (see also the next section).

In resume, initial equations of motion (2) for singular theory have been rewritten in special coordinates (19) on the configurationvelocity space. Motivation for making of the change was to find normal form of differential equations (17). It turns out to be sufficient to transform the variables $v^{i}$ only, defining the new variables $p_{i}\left(v^{j}\right)$ in accordance with Eq.(22). One concludes that representation of singular Lagrangian dynamics in the normal form (25)-(27) do not implies any extension of the configuration-velocity space. In particular, the primary Dirac constraints do not appear in the formulation. We have demonstrated also that the Dirac second stage system $\left\{\Phi_{\alpha}, H\right\}=0$ represents part of Lagrangian equations of motion (18), rewritten in the coordinates $\left(q^{A}, p_{i}, v^{\alpha}\right)$. By construction, the systems (2) and (25)-(27) are equivalent. It would be interesting to analyze local symmetries $[4,3]$ in this framework, as well as to compare the reduced scheme with other modifications of the Dirac procedure $[5,6]$. 


\section{Equivalence with the Dirac procedure}

We discuss here relation among the system (25)-(27) and the standard Hamiltonian formulation (6). Starting from (25)-(27), let us define an equivalent system on extended space with the coordinates $\left(q^{A}, p_{j}, v^{\alpha}, p_{\alpha}\right)$. By definition, $\left(q^{A}, p_{j}, v^{\alpha}\right)$-sector obeys the Eqs.(25)(27), while for $p_{\alpha}$ one writes the primary Dirac constraints

$$
\Phi_{\alpha} \equiv p_{\alpha}-f_{\alpha}\left(q^{A}, p_{j}\right)=0 .
$$

Then Eq.(27) can be rewritten identically in the form $\left\{\Phi_{\alpha}, H\right\}=0$, while the following equation:

$$
\dot{p}_{\alpha}=-\frac{\partial H}{\partial q^{\alpha}}
$$

is a consequence of Eqs.(25) -(27), (30), and thus can be added to this system. After that, Eq.(27) turns out to be consequence of others, and can be omitted. The resulting system (25), (26), (30), (31) coincides exactly with the Hamiltonian formulation (6) (modulo of notations).

This discussion reveals that the only role, playing by $p_{\alpha}$ in the Dirac formulation, is to represent equations of motion in completely symmetric form, with the Poisson bracket defined in relation to all variables $q^{A}, p_{A}$. The momenta $p_{\alpha}$ are, in fact, "Hamiltonian ghosts" of singular theory. The symmetrization seems to be unnecessary ingredient of this stage. Further stages of the Dirac procedure (on revealing of higher-stage constraints) do not require the symmetric form of equations. It may be more convenient to make further steps by using of the reduced formulation, and then to symmetrize the resulting equations.

\section{Conclusion}

In this work it was demonstrated that Hamiltonian formulation for singular theory (11)-(3) can be considered as the first order Lagrangian formulation (9) on the space $\left(q^{A}, v^{i}, v^{\alpha}\right)$, further rewritten in special coordinates $\left(q^{A}, p_{i}, v^{\alpha}\right)$ of this space. The corresponding change of variables was find from the requirement that equations (9) acquire normal form in the new coordinate system. The transformation $v^{i}\left(q^{A}, p_{j}, v^{\alpha}\right)$, defined implicitly by Eq.(22), turns out to 
be sufficient condition to this end. Search for the normal form do not implies use of an additional variables, as well as the primary Dirac constraints. The resulting formulation (25) -(27) involves algebraic equations (27) linear on $v^{\alpha}$. They coincide with the second stage equations of the Dirac procedure: $\left\{\Phi_{\alpha}, H\right\}=0$. The system (25) - (27) and the standard one (6) are equivalent.

For the scheme presented one writes, schematically

$$
q^{A} \rightarrow\left(q^{A}, v^{i}, v^{\alpha}\right) \leftrightarrow\left(q^{A}, p_{i}, v^{\alpha}\right) .
$$

It can be compared with the standard approach: according to [2], Hamiltonian formalism involves more variables

$$
q^{A} \rightarrow\left(q^{A}, v^{i}, v^{\alpha}\right) \rightarrow\left(q^{A}, v^{i}, v^{\alpha}, p_{i}, p_{\alpha}\right) .
$$

Dynamics of $\left(q^{A}, v^{A}\right)$-sector is governed by the Lagrangian first order equations (9), while $p_{A}=\frac{\partial \bar{L}}{\partial v^{A}}$.

\section{Acknowledgments}

Author would like to thank the Brazilian foundations $\mathrm{CNPq}$ and FAPEMIG for financial support.

\section{Appendix}

We present here properties of the function $v^{i}\left(q^{A}, p_{j}, v^{\alpha}\right)$ defined implicitly by Eq.(22), as well as properties of the Lagrangian $L$ in terms of the coordinates (19): $\left.L\left(q^{A}, p_{j}, v^{\alpha}\right) \equiv \bar{L}\left(q^{A}, v^{i}, v^{\alpha}\right)\right|_{v^{i}}$. In particular, both functions turn out to be at most linear on $v^{\alpha}$ in any singular theory (11)-(3).

Derivatives of the identity $\left.p_{i} \equiv \frac{\partial \bar{L}\left(q^{A}, v^{i}, v^{\alpha}\right)}{\partial v^{i}}\right|_{v^{i}\left(q^{A}, p_{j}, v^{\alpha}\right)}$ give the relations (see our notations (11), (12))

$$
\frac{\partial v^{i}}{\partial p_{j}}=\tilde{M}^{i j}, \quad \frac{\partial v^{i}}{\partial v^{\alpha}}=-\tilde{M}^{i j} M_{j \alpha}, \quad \frac{\partial v^{i}}{\partial q^{A}}=-\left.\tilde{M}^{i j} \frac{\partial^{2} \bar{L}}{\partial v^{j} \partial q^{A}}\right|_{v^{i}}
$$

Then the identity (13) acquires the form $\frac{\partial}{\partial v^{\alpha}}\left(\left.\frac{\partial \bar{L}}{\partial v^{\beta}}\right|_{v^{i}}\right)=0$, so the quantity

$$
\left.f_{\alpha}\left(q^{A}, p_{j}\right) \equiv \frac{\partial \bar{L}}{\partial v^{\alpha}}\right|_{v^{i}}
$$


does not depend on $v^{\alpha}$. One finds $\frac{\partial f_{\alpha}\left(q^{A}, p_{j}\right)}{\partial p_{i}}=\tilde{M}^{i j} M_{j \alpha}$, so the quantity $\tilde{M}^{i j} M_{j \alpha}$ does not depend on $v^{\alpha}$ also. Moreover, Eq.(35) can be identically rewritten in terms of $L$

$$
f_{\alpha}\left(q^{A}, p_{j}\right)=-\frac{\partial}{\partial v^{\alpha}}\left(p_{i} v^{i}-L\right)
$$

which implies that the quantity

$$
H_{R}\left(q^{A}, p_{j}, v^{\alpha}\right) \equiv\left(p_{i} v^{i}-L\right),
$$

is at most linear on $v^{\alpha}$. Integral of Eq.(36) gives the following expression for $H_{R}$

$$
H_{R}\left(q^{A}, p_{j}, v^{\alpha}\right)=H_{0}\left(q^{A}, p_{j}\right)-v^{\alpha} f_{\alpha}\left(q^{A}, p_{j}\right),
$$

where $H_{0}$ does not depend on $v^{\alpha}$. Expression for this quantity in terms of initial Lagrangian can be obtained by comparison of Eqs. (37), (38)

$$
H_{0}\left(q^{A}, p_{j}\right)=\left.\left(\frac{\partial \bar{L}}{\partial v^{A}} v^{A}-\bar{L}\right)\right|_{v^{i}} .
$$

Note that it coincides exactly with the Hamiltonian $H_{0}$ (8) of the standard scheme. From Eq.(37) one obtains useful relations

$$
\frac{\partial H_{R}}{\partial p_{i}}=v^{i}, \quad \frac{\partial H_{R}}{\partial q^{A}}=-\left.\frac{\partial \bar{L}}{\partial q^{A}}\right|_{v^{i}}, \quad \frac{\partial H_{R}}{\partial v^{\alpha}}=-f_{\alpha}\left(q^{A}, p_{j}\right) .
$$

In particular, the first equation states that our function $v^{i}$ is at most linear on $v^{\alpha}$ and has the representation

$$
v^{i}\left(q^{A}, p_{j}, v^{\alpha}\right)=\frac{\partial H_{0}}{\partial p_{i}}-\frac{\partial f_{\alpha}}{\partial p_{i}} v^{\alpha} .
$$

It implies (see Eq.(34) ) that the matrix $\tilde{M}^{i j}$ is at most linear on $v^{\alpha}$, and has the representation $\tilde{M}^{i j}=\frac{\partial^{2} H_{R}}{\partial p_{i} \partial p_{j}}$. At last, Eqs.(37), (38), (41) imply that $L$ is at most linear on $v^{\alpha}$ and has the representation

$$
L\left(q^{A}, p_{j}, v^{\alpha}\right)=p_{i} \frac{\partial H_{R}}{\partial p_{i}}-H_{R}
$$

In resume, we have demonstrated, in fact, that in the coordinates $\left(q^{a}, p_{i}, v^{\alpha}\right)$ all quantities of the first order Lagrangian formulation (9) -(10) can be rewritten in terms of the functions $H_{0}\left(q^{A}, p_{j}\right), f_{\alpha}\left(q^{A}, p_{j}\right)$. 


\section{References}

[1] P.A.M. Dirac, Can. J. Math. 2 (1950) 129; Lectures on Quantum Mechanics (Yeshiva Univ., New York, 1964).

[2] D. M. Gitman and I. V. Tyutin, Quantization of Fields with Constraints (Berlin: Springer-Verlag, 1990).

[3] A. A. Deriglazov, and K.E. Evdokimov, Int. J. Mod. Phys. A 15 (2000) 4045 hep-th/9912179; A. A. Deriglazov, Note on Lagrangian and Hamiltonian symmetries, hep-th/9412244

[4] V. A. Borovkov and I. V. Tyutin, Physics of Atomic Nuclei 61 (1998) 1603; Physics of Atomic Nuclei 62 (1999) 1070; D. M. Gitman and I. V. Tyutin, Symmetries and physical functions in general gauge theory, hep-th/0503218.

[5] J. Barcelos-Neto and C. Wotzasek, Mod. Phys. Lett. A7 (1992) 1737; C. Neves, W. Oliveira, D. C. Rodrigues and C. Wotzasek, Phys. Rev. D69 (2004) 045016; E. M. C. Abreu, C. Neves and W. Oliveira, Noncommutativity from the symplectic point of view, hep-th/0411108.

[6] M.N. Stoilov, Canonical approach to Lagrange multipliers, hep-th/0504099. 